\title{
Enhanced Endogenous Insulin Secretion after Treatment with Monocomponent Insulin
}

\author{
YASUAKI FUKUMOTO, KIKUO ICHIHARA \\ AND SEIICHIRo TARUI \\ Second Department of Internal Medicine, Osaka University \\ Medical School, Fukushima-ku, Osaka 553, Japan
}

\begin{abstract}
Synopsis
Eight maturity-onset diabetic patients who had no infections, liver diseases or other endocrine diseases which might affect carbohydrate metabolism were treated with monocomponent lente insulin, which is known to have little antigenicity, until blood sugar was controlled. The diurnal values of the blood sugar (BS) and immunoreactive insulin (IRI) were obtained before treatment and on the third day after the withdrawal of insulin treatment. One patient was dropped from the study because of insulin antibody formation. The total integrated IRI area in the diurnal profile was increased from $98.9 \pm 23.7$ to $186.1 \pm 37.7 \mu \mathrm{U} \cdot \mathrm{h} / \mathrm{ml}(\mathrm{p}<0.05)$, while the total blood sugar area was decreased from $7,500 \pm 1,200$ to $5,510 \pm 1,214 \mathrm{mg} \cdot \mathrm{h} \%(\mathrm{p}<0.05)$. Oral Glucose Tolerance Test on the fourth day after the withdrawal of insulin treatment showed increased IRI response compared to pretreatment.

It is concluded that endogenous insulin secretion could be increased in the diabetic patient by the metabolic control with exogenous insulin injections.
\end{abstract}

When overt diabetic patients are treated with insulin, it is often observed that the dosage of insulin required to control the blood sugar is reduced or insulin may no longer be necessary and can be replaced by oral agents or even a diet alone will be sufficient. There will be several reasons to explain this fact, such as elimination of infections, ketosis, stress and other complications, which could cause deterioration of diabetes. It is also possible that endogenous insulin secretion is increased by insulin treatment.

Usually insulin antibodies are formed by the treatment with conventional insulin preparations (Anderson, 1972), which makes it practically impossible to analyze the capacity of endogenous insulin secretion by measuring circulating IRI levels because circulating

Received April 2, 1977. insulin antibodies interfere with the insulin immunoassay system. In order to see the influence of exogenous insulin on endogenous insulin secretion, bonito fish insulin was used in some institutes (Odagiri, 1971; Toyota et al., 1972) to avoid this immunological problem and some assessed indirectly by C-peptide assay (Block, et al., 1973). However, if there were insulin preparations in clinical use which do not form insulin antibodies, it should be possible to assess directly how the treatment with insulin administration influenced the endogenous insulin secretion.

As we have obtained monocomponent (MC) insulin, which has been shown to form far fewer antibodies than the conventional preparations (Schlichtkrull et al., 1972), we have explored the possibility of increased endogenous insulin secretion by insulin treatment. 


\section{Materials and Methods}

Four males and four females maturity-onset diabetic patients aged from 39 to 74 years, mean $52.9 \pm 5.3$ (S.E.) were studied. It was two weeks to fifteen months since they had symptoms of diabetes and none of them had had insulin treatment before. Their height ranged from 153 to $168 \mathrm{~cm}$, mean 158.3 $\pm 2.4 \mathrm{~cm}$, weight 41 to $76 \mathrm{~kg}$, mean $56.4 \pm 5.0 \mathrm{~kg}$, and fasting blood sugar (FBS) from 220 to $450 \mathrm{mg} \%$, mean $313 \pm 31.2 \mathrm{mg} \%$. None had infections, liver diseases or endocrine diseases which might affect carbohydrate metabolism.

They were all admitted to hospital and the diet was standardized to $25 \mathrm{cal}$ per day per $\mathrm{kg}$ ideal body weight according to the Jones method, height $(\mathrm{cm})-$ $100-\frac{\text { height }-150}{4}$. No food intake was allowed other than meals, which were provided three times a day at 0800,1200 and $1700 \mathrm{hr}$. On admission, the blood was taken eight times at $0600 \mathrm{hr}$, just before and $90 \mathrm{~min}$ after each meal and at $2400 \mathrm{hr}$, to obtain a diurnal profile of blood sugar (BS) and serum immunoreactive insulin (IRI). A $100 \mathrm{~g}$ oral glucose tolerance test was performed on the following morning; then the treatment with daily injections of porcine monocomponent (MC) insulin (Monotard, Novo) was initiated. When FBS and urine sugar became less than $150 \mathrm{mg} \%$ and $10 \mathrm{~g} / 24 \mathrm{hr}$, respectively, we judged that the diabetes was controlled, and insulin injections were discontinued for the reexamination of endogenous insulin secretion while the diet remained the same. MC insulin was given for from 14 to 32 days, average 22.6 days, and blood sugar became $134.5 \pm 5.6 \mathrm{mg} \%$, ranging from 116 to $151 \mathrm{mg} \%$. A diurnal profile of BS and IRI was obtained on the third day after insulin was discontinued and the second OGTT was performed on the 4th day.

Blood sugar was measured by the Hoffman method and serum IRI was assayed by the solid phase method (Phadebas Kit). The total blood sugar area and incremental IRI area in the diurnal profile were calculated respectively as follows, (eight consecutive values of $\mathrm{BS}$ and IRI are expressed as $\mathrm{A}, \mathrm{B}, \ldots$ and $\left.\mathrm{A}^{\prime}, \mathrm{B}^{\prime} \ldots\right)$.

Total BS area: $\quad 2 \cdot \frac{\mathrm{A}+\mathrm{B}}{2}+1 \frac{1}{2} \cdot \frac{\mathrm{B}+\mathrm{C}}{2}+2 \frac{1}{2}$. $\frac{\mathrm{C}+\mathrm{D}}{2}+1 \frac{1}{2} \cdot \frac{\mathrm{D}+\mathrm{E}}{2}+3 \frac{1}{2} \cdot \frac{\mathrm{E}+\mathrm{F}}{2}+1 \frac{1}{2} \cdot \frac{\mathrm{F}+\mathrm{G}}{2}+5 \frac{1}{2}$ . $\frac{\mathrm{G}+\mathrm{H}}{2}=\mathrm{A}+1.75 \times \mathrm{B}+2 \times(\mathrm{C}+\mathrm{D})+2.5 \times(\mathrm{E}+\mathrm{F})+$ $3.5 \times \mathrm{G}+2.75 \times \mathrm{H}$

Incremental IRI area: $2 \cdot \frac{\mathbf{A}^{\prime}+\mathbf{B}^{\prime}}{2}+1 \frac{1}{2} \cdot \frac{\mathbf{B}^{\prime}+\mathbf{C}^{\prime}}{2}$ $+2 \frac{1}{2} \cdot \frac{C^{\prime}+D^{\prime}}{2}+1 \frac{1}{2} \cdot \frac{D^{\prime}+E^{\prime}}{2}+3 \frac{1}{2} \cdot \frac{E^{\prime}+F^{\prime}}{2}+1 \frac{1}{2}$. $\frac{\mathrm{F}^{\prime}+\mathrm{G}^{\prime}}{2}+5 \frac{1}{2} \cdot \frac{\mathrm{G}^{\prime}+\mathrm{H}^{\prime}}{2}-18 \cdot \frac{\mathrm{A}^{\prime}+\mathrm{B}^{\prime}}{2}=\mathrm{A}^{\prime}+1.75 \times \mathrm{B}^{\prime}+$
$2 \times\left(\mathrm{C}^{\prime}+\mathrm{D}^{\prime}\right)+2.5 \times\left(\mathrm{E}^{\prime}+\mathrm{F}^{\prime}\right)+13.5 \times \mathrm{G}^{\prime}+2.75 \times \mathrm{H}^{\prime}-18$ $\times \frac{\mathrm{A}^{\prime}+\mathrm{B}^{\prime}}{2}$ Similarly, the blood sugar (IRI) area on OGTT was calculated as follows (six consecutive values expressed as $a, b, c, \ldots \ldots)$ :

$\frac{1}{2} \cdot \frac{a+b}{2}+\frac{1}{2} \cdot \frac{b+c}{2}+\frac{1}{2} \cdot \frac{c+d}{2}+\frac{1}{2} \cdot \frac{d+e}{2}+1 \cdot \frac{e+f}{2}$

$=\frac{1}{2}\{0.5 \times \mathrm{a}+(\mathrm{b}+\mathrm{c}+\mathrm{d}+\mathrm{f})+1.5 \times \mathrm{e}\}$.

Insulin antibodies were searched for by ${ }^{125}$ I-Insulin binding rate; $0.1 \mathrm{ml}$ of fasting serum of each patient and $4 \mu \mathrm{U}$ of ${ }^{125} \mathrm{I}$-insulin were incubated for $24 \mathrm{hr}$ at $4^{\circ} \mathrm{C}$, the antibody-bound insulin was separated by dextran coated charcoal (Herbert et al., 1965), and the ratio of radioactivity of antibody-bound insulin to the total radioactivity in a tube was calculated.

For the statistical evaluation, Wilcoxon's method was used (Wilcoxon, 1945).

\section{Results}

\section{Insulin antibody formation}

To detect circulating insulin antibodies, we measured ${ }^{125} \mathrm{I}$-insulin binding rate of the fasting serum before and after MC insulin treatment on each patient (Table 1). Insulin antibodies were not formed in seven patients in this study. As Case 8 showed a high ${ }^{125}$ I-insulin binding rate after treatment, we considered that this patient formed insulin antibodies and was, therefore, dropped from our study.

Changes in a diurnal profile of blood sugar and serum IRI by treatment

After treatment, fasting blood sugar decreased significantly from the pretreatment value, $313 \pm 31 \mathrm{mg} \%$ (mean \pm S.E.) to $221 \pm$ $36 \mathrm{mg} \%(\mathrm{p}<0.05)$, and the blood sugar value at any point in diurnal profile was lower than before treatment. The area below the curve of diurnal profile, which we call "total blood sugar area", decreased significantly from $7,500 \pm 1,220$ to $5,511 \pm 1,214$ $\mathrm{mg} \cdot \mathrm{h} \%(\mathrm{p}<0.05)$ (Fig. 1).

The diurnal profile of serum IRI concentrations before treatment shows a low flat curve, and after treatment serum insulin levels have increased significantly, and the profile shows rhythmic changes by meals, 
Table 1. Insulin binding rate of the serum in patients treated with MC Insulin and conventional insulin preparations.

\begin{tabular}{|c|c|c|}
\hline \multirow[t]{2}{*}{ Cases } & \multicolumn{2}{|c|}{ Insulin binding rate } \\
\hline & Before & After \\
\hline \multicolumn{3}{|c|}{ Patients treated with } \\
\hline \multicolumn{3}{|c|}{ MC Insulin } \\
\hline 1. & $2.6 \%$ & $0.7 \%$ \\
\hline 2. & 2.4 & 4.4 \\
\hline 3. & 0.5 & -1.1 \\
\hline 4. & 0.3 & 0.3 \\
\hline 5. & 2.1 & 1.5 \\
\hline 6. & -1.4 & -2.1 \\
\hline 7. & 0.5 & -1.6 \\
\hline 8. & 0 & 13.1 \\
\hline \multicolumn{3}{|c|}{ Non-diabetic Control } \\
\hline 1. & & 3.3 \\
\hline 2. & & -0.9 \\
\hline 3. & & -2.4 \\
\hline \multicolumn{3}{|c|}{$\begin{array}{l}\text { Patients treated with } \\
\text { conventional preparations }\end{array}$} \\
\hline 1. & & 42.5 \\
\hline 2. & & 24.0 \\
\hline 3. & & 26.4 \\
\hline 4. & & 42.0 \\
\hline 5. & & 16.0 \\
\hline
\end{tabular}

The mean value of the three non-diabetic control was used as "control", $(0 \%)$. suggesting the increased responsiveness of endogenous secretion to the stimulation of food intake (Fig. 2).

The incremental IRI area, the area above the fasting level (mean value at 0600 and $0800 \mathrm{hr}$ ) and below the diurnal curve, has increased by $88 \%$ from the pretreatment value $98.9 \pm 23.7 \mu \mathrm{U} \cdot \mathrm{h} / \mathrm{m} l$ to $186.1 \pm 37.7$ $\mu \mathrm{U} \cdot \mathrm{h} / \mathrm{m} l(\mathrm{p}<0.05)$ after treatment (Fig. 2).

Changes in $100 \mathrm{~g}$ oral glucose tolerance test (Fig. 3)

The blood sugar at any point and total blood sugar area after treatment are lower than before treatment, though there was no statistical significance. However, the serum IRI response was significantly higher in the early stage up to $90 \mathrm{~min}$ after glucose loading $(\mathrm{p}<0.05)$.

The relation between weight change and insulin secretion

The body weight of each subject was checked during this study period. One patient with a 15-month history of diabetes
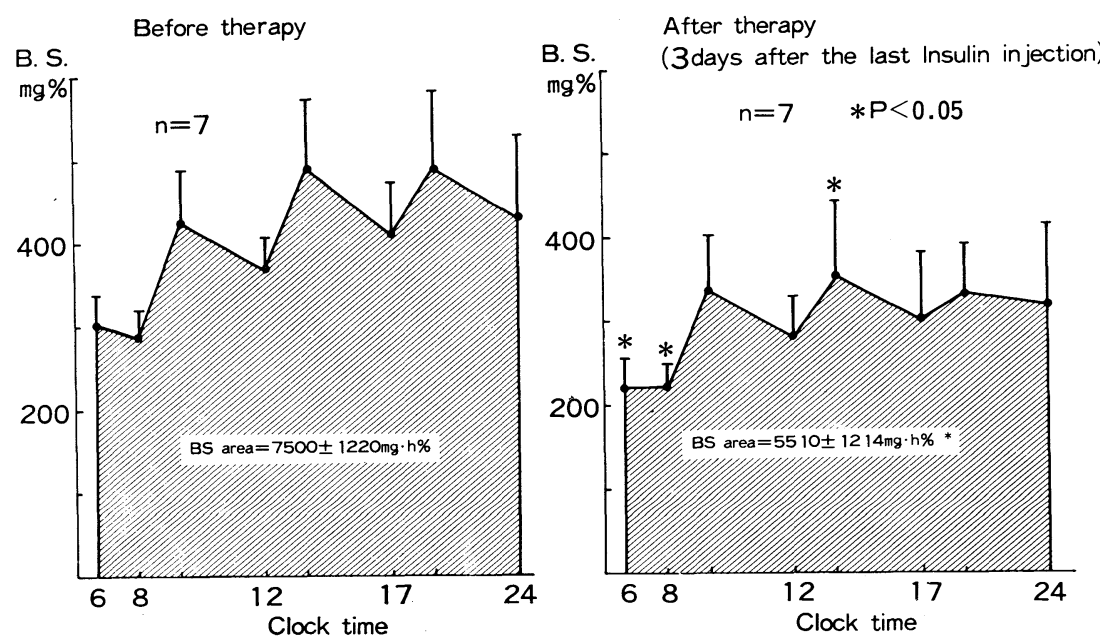

Fig. 1. Changes of the diurnal profile of blood sugar and blood sugar area by insulin therapy. Blood sugar was measured at $0600 \mathrm{hr}$, just before and $90 \mathrm{~min}$ after each meal and at $2400 \mathrm{hr}$. Vertical bars show the standard error (S.E.)

* means statistical significance $(\mathrm{p}<0.05)$ comparing each corresponding time before and after therapy. 
Before therapy

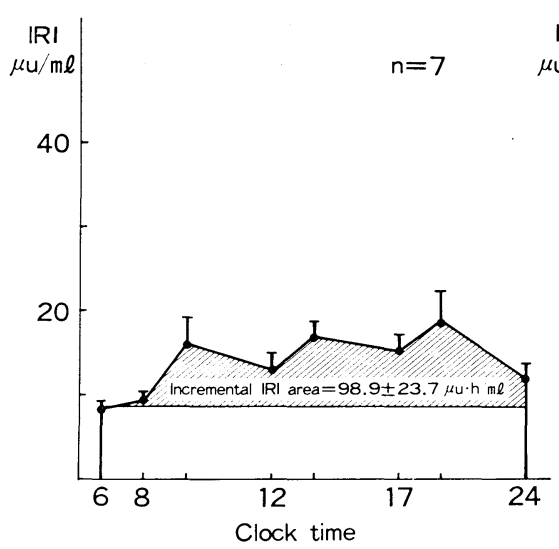

After therapy

( 3 days after the last insulin injection)

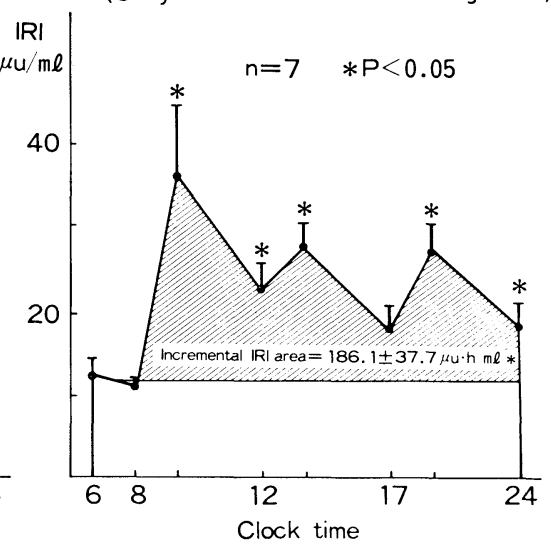

Fig. 2. Diurnal profile of serum IRI levels. Note the increased IRI levels responding to meals after insulin therapy.
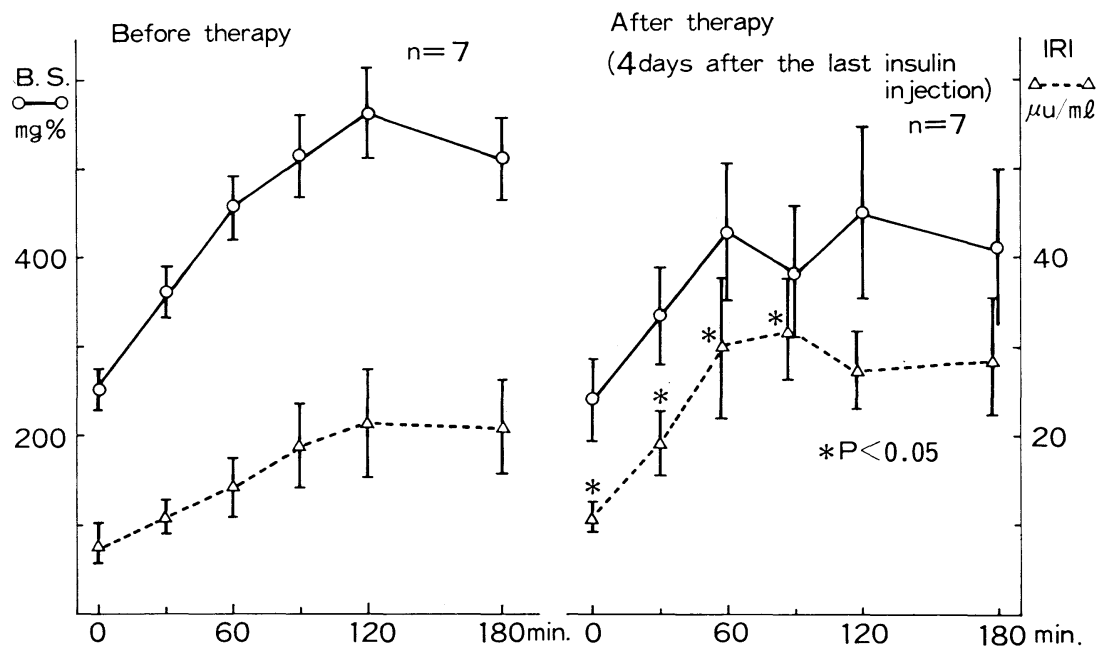

Fig. 3. Effect of insulin therapy on oral glucose tolerance test.

gained weight by $9.3 \%$ from 40.6 to $44.4 \mathrm{~kg}$ (height, $154 \mathrm{~cm}$ ) in 26 days of successful insulin therapy, FBS having decreased from 450 to $150 \mathrm{mg} \%$. However, in three days without insulin, FBS came up to $348 \mathrm{mg} \%$ and the diurnal profile of IRI did not show any improvement from the initial low, flat curve $(6 \mu \mathrm{U} / \mathrm{m} l$ at $0600 \mathrm{hr}$ and maximum $13 \mu \mathrm{U} / \mathrm{m} l 90 \mathrm{~min}$ after breakfast).

In the other six patients, the weight change was small (within $\pm 2 \%$ ), and we could not find any correlation between the weight change and the insulin secretion. 


\section{Discussion}

Our study showed that serum IRI levels increased with $\mathrm{MC}$ insulin treatment. As the duration of action of MC lente insulin is less than $24 \mathrm{hr}$ (Kasama et al., 1975), it is hard to believe that exogenously administered insulin is still circulating in the blood stream 3 days after it is discontinued. We can therefore attribute this increment of IRI after treatment to the endogenously secreted insulin. Even if there should be the residual, exogenously administered insulin in the blood stream, we can still conclude that the endogenously secreted insulin has increased after treatment, because IRI levels are rhythmically higer after each meal and also the incremental area from the fasting level has increased.

We consider that the injured B-cells are affected indirectly by an improved general metabolic state with administered insulin and the residual reversibly injured parts are regenerated. In mild diabetic patients diet therapy was demonstrated to augment the endogenous insulin secretion (Ichihara et al., 1975). But with this degree of diabetes in our subjects, with markedly high blood sugar and low IRI levels, it is difficult to expect much improvement in the metabolic control by diet therapy alone. This was supported by the result shown three days after insulin withdrawal by the rapid deterioration in FBS from $134 \pm 5$ to $221 \pm$ $32 \mathrm{mg} \%$.

Therefore, we conclude that the increased endogenous insulin secretion after this study was achieved mainly by insulin therapy, though the minor role of concomitant diet therapy had to be reckoned with, too.

Compared to the conventional insulin preparations, highly purified MC insulin and single peak insulin (Lilly) are shown to have very little antigenicity in man or rabbits (Root et al., 1972; Schlichtkrull et al., 1972; Czyzyk et al., 1974). There were no detectable insulin antibodies in our seven cases when examined by ${ }^{125} \mathrm{I}$-insulin binding rate. This is partly due to the experimental design, in which the insulin doses were small and the duration of insulin administration was relatively short. This speculation may be supported by Case 8 (Table 1), who was administered with MC Insulin in larger doses (up to 68 units a day) for a longer period (40 days) than the other seven cases and developed insulin antibodies.

Five patients in this study were successfully treated with oral hypoglycemic agents (glibenclamide) thereafter, and two patients needed further insulin therapy to be kept under good control. As the latter were suffering from diabetes for longer periods (12 and 15 months respectively) than the others, (2, 3, 3, 6 weeks and 5 months respectively) pathological changes of the islet cells must have been advanced to an irreversible degree.

\section{References}

Anderson, O. (1972). Acta Endocrinol. (Kbh) 71, 126. Block, M., R. L. Rosenfield, M. E. Mako, D. F. Steiner aud A. H. Rubenstein (1973). New Engl. J. Med. 288, 1144.

Czyzyk, A., J. Lawecki, H. Rogela, E. Miedzinska and A. Popik-Hankewicz (1974). Diabetes 10, 233.

Herbert, V., K. S. Lau, C. W. Gottlib and S. J. Bleicherl(1965). J. Clin. Endocrinol. Metab. 25, 1375. Ichihara, K., K. Shima, K. Nonaka and S. Tarui (1975). Endocrinol. Japon. 22, 399.

Kasama, T., H. Nakajima, S. Yonezawa, T. Iiyama, M. Uchida and S. Nakagawa (1975). J. Japan Diab. Soc. 18, 29.

Odagiri, R. (1971). ibid. 14, 245.

Root, M. A., R. E. Chance and J. A. Galloway (1972). Diabetes 21 (Suppl. 2), 657.

Schlichtkrull, J., J. Brange, Aa. H. Christiansen, O. Hallund, L. G. Heding and K. H. Jorgensen (1922). ibid. 21 (Suppl. 2), 649.

Toyota, T., S. Sato, A. Ohneda and Y. Goto (1972). Tohoku J. Exp. Med. 107, 167.

Wilcoxon, F. (1945). Biometrics 1, 80. 